\title{
Impact of methanol intoxication on the human electrocardiogram
}

\author{
Zardasht Jaff $^{1}$, William F. McIntyre ${ }^{1}$, Payam Yazdan-Ashoori ${ }^{2}$, Adrian Baranchuk ${ }^{1}$ \\ ${ }^{1}$ Division of Cardiology, Queen's University, Kingston, Ontario, Canada \\ ${ }^{2}$ McMaster University, Internal Medicine, Hamilton, Ontario, Canada
}

\begin{abstract}
Background: Methanol is a common commercial compound that can lead to significant morbidity and mortality with high levels of exposure. The purpose of this study was to describe electrocardiographic (ECG) changes associated with methanol intoxication.

Methods: A retrospective chart review was conducted with data from Kingston General Hospital collected between 2006 and 2011. Patient data, including demographics, medications, and laboratory data were recorded. Twelve-lead ECGs were obtained and changes were noted in relation to timing and extent of methanol intoxication.

Results: Nine patients with a mean age of 45 years were analyzed. All patients ingested methanol orally and presented to hospital between $<1$ to $25 \mathrm{~h}$ after ingestion. The mean plasma methanol concentration on admission was $49.8 \mathrm{mmol} / \mathrm{L}$. A lower $p H$ and higher plasma methanol concentration were associated with multiple ECG changes. On admission, ECG changes included sinus tachycardia (44\%), PR prolongation (11\%), QT prolongation (22\%) and non-specific T-wave changes (66\%). One patient developed a type-1 Brugada ECG pattern. During their course in hospital, 7 patients required dialysis, 3 required mechanical ventilation, 3 developed visual impairment, and 1 died. All ECG changes normalized while in hospital.

Conclusions: Methanol intoxication can lead to several ECG changes with sinus tachycardia and non-specific T-wave changes being the most common. These changes were more prominent in cases of severe acidosis. (Cardiol J 2014; 21, 2: 170-175)
\end{abstract}

Key words: methanol intoxication, overdose, electrocardiogram

\section{Introduction}

Methanol is a wood alcohol that is a clear and colorless liquid with a mild alcoholic odor. It is an additive found in many commercial products including antifreeze, windshield cleaner, paint thinner and rubbing alcohol [1]. Methanol intoxication can result in significant morbidity and mortality in the emergency patient population [2,3]. Approximately 2,283 possible methanol exposures were reported to poison control centers in the United States in 2007 [4]. Although routes of entry into the body include inhalation and dermal absorption, most methanol intoxications result from oral ingestion [5]. After ingestion, methanol is quickly metabolized to formaldehyde and subsequently oxidized to formic acid. Formic acid is responsible for most of the known adverse effects of methanol ingestion, including metabolic acidosis, ocular toxicity, and neurological disturbances such as coma and convul-

Address for correspondence: Zardasht Jaff, MBChB, PGY3, Department of Internal Medicine, Queen's University, Kingston General Hospital, 76 Stuart Street Kingston, ON K7L 2V7, Ontario, Canada, tel: 001-613-985-2511, e-mail: gzardash@gmail.com

Received: 30.03.2013 Accepted: 19.04.2013 
Table 1. Patient characteristics.

\begin{tabular}{ccccccc}
\hline Patient Gender & Age & $\begin{array}{c}\text { Time between } \\
\text { ingestion to } \\
\text { hospitalization [h] }\end{array}$ & $\begin{array}{c}\text { Route of } \\
\text { administration }\end{array}$ & $\begin{array}{c}\text { Source of } \\
\text { methanol } \\
\text { intoxication }\end{array}$ & $\begin{array}{c}\text { Initial methanol } \\
\text { concentration } \\
\text { [mmol/L] }\end{array}$ \\
\hline 1 & Male & 46 & 6.5 & Oral & Windshield washer & 69.8 \\
2 & Female & 45 & $<1$ & Oral & Windshield washer & 35.8 \\
3 & Female & 29 & $<1$ & Oral & Fuels & 11.3 \\
4 & Male & 49 & 25 & Oral & Windshield washer, & 88.6 \\
& & & & & gasoline antifreeze, & \\
5 & Male & 24 & 25 & Oral & Windshield washer & 31.4 \\
6 & Male & 42 & 25 & Oral & Windshield washer & 128 \\
7 & Male & 45 & $<1$ & Oral & None reported & 7.4 \\
8 & Female & 64 & 24 & Oral & None reported & 24.5 \\
9 & Female & 84 & $<1$ & Oral & None reported & 51.5 \\
\hline
\end{tabular}

sions [1]. In severe cases, methanol intoxication can progress to multi-organ failure and death.

Currently, there are limited data on the ECG manifestations associated with methanol intoxication. Previous reports have documented changes in axis, non-specific T-wave changes, minor changes in QRS complexes and prolongation of the $\mathrm{QT}_{\mathrm{c}}$ interval [6-8].

Given the paucity of reported cases in the literature, no systematic ECG analysis in this patient population has been published to date. Additionally, no correlation between ECG changes and metabolic acidosis has been reported. The aim of this study is to report all methanol intoxications occurring in a single center and to describe their impact on the ECG.

\section{Methods}

\section{Study description}

A retrospective study was conducted among patients presenting with methanol intoxication to determine ECG changes and their relation to methanol levels, $\mathrm{pH}$, anion and osmolar gaps. Methanol intoxication was defined as a plasma methanol concentration greater than $6.2 \mathrm{mmol} / \mathrm{L}$ [9].

\section{Study population}

Patients included in this study were $\geq 18$ years of age with at least one plasma methanol concentration greater than $6.2 \mathrm{mmol} / \mathrm{L}$ obtained during the course of hospitalization at Kingston General Hospital between 2006 and 2011. The electronic charts were reviewed and baseline clinical information including demographics, medical history and medications were obtained. Patients with established cardiovascular disease were excluded to minimize potential confounding of ECG changes.
Exclusion criteria included patients with a history of myocardial infarction, dilated ischemic or non-ischemic cardiomyopathy, persistent or permanent atrial fibrillation or atrial flutter, and patients who underwent percutaneous coronary intervention or aortocoronary bypass within the past 6 months or had an intracardiac device.

\section{Data analysis}

Available ECGs which obtained closest to peak methanol concentrations were analyzed for abnormalities including non-sinus rhythm, changes in waveform amplitude or shape, ST-segment changes, T-wave changes, and/or changes in PR, $\mathrm{QRS}$ and $\mathrm{QT}_{\mathrm{c}}$ intervals. Statistical analysis of demographic and laboratory data was performed by determining their mean and standard deviations. A cardiac electrophysiologist (AB) reviewed available ECGs both during the intoxication and after resolution for evidence of reversibility. This study was approved by the Queen's Health Sciences Research Ethics Board.

\section{Results}

Clinical characteristics of the study population are summarized in Table 1 . Nine patients with a mean age of 45 years were included in the analysis. All patients ingested methanol orally with windshield washer fluid being the most common source. All patients presented to hospital between $<1$ to $25 \mathrm{~h}$ after ingestion. The mean plasma methanol concentration on admission was $49.8 \mathrm{mmol} / \mathrm{L}$. The mean $\mathrm{pH}$, anion gap and osmolar gap on admission were $7.20,20.45$ and 48.6, respectively (Table 2 ).

ECG changes are summarized in (Table 3 ). Seven of the 9 patients showed definite ECG changes. 
Table 2. Laboratory results.

\begin{tabular}{lcccccccccccc}
\hline Patient & $\mathbf{p H}$ & $\mathbf{p C O}_{2}$ & $\mathrm{HCO}_{3}$ & $\mathrm{Na}$ & $\mathbf{K}$ & $\mathrm{Cl}$ & $\mathrm{SCr}$ & Urea & $\mathrm{AG}$ & OG & $\begin{array}{c}\text { Max } \\
\text { CK }\end{array}$ & $\begin{array}{c}\text { Max } \\
\text { troponin }\end{array}$ \\
\hline 1 & 7.01 & 36 & 9 & 143 & 4.4 & 109 & 120 & 3.8 & 25.0 & 85.6 & 274 & 0.279 \\
2 & 7.09 & 26 & 8 & 139 & 4.5 & 98 & 56 & 4.0 & 33.0 & 0.5 & NA & NA \\
3 & 7.42 & 44 & 28 & 139 & 3.9 & 102 & 1553 & 3.7 & 9 & 21.1 & 120 & 0.032 \\
4 & 7.16 & 15.5 & 5.3 & 134 & 5.8 & 100 & 93 & 2.6 & 28.7 & 111.4 & 103 & 0.016 \\
5 & 7.19 & 36.6 & 11.9 & 130 & 5.0 & 98 & 98 & 4.3 & 20.1 & 53.5 & 300 & 0.027 \\
6 & 6.91 & 26.4 & 5.1 & 133 & 5.9 & 92 & 166 & 12.2 & 35.9 & 172.5 & NA & 0.021 \\
7 & 7.36 & 49 & 26.7 & 137 & 4.1 & 104 & 78 & 5.0 & 6.3 & 16.0 & NA & NA \\
8 & 7.30 & 101 & 48.5 & 167 & 3.0 & 110 & 69 & 21.0 & 8.5 & 19.9 & NA & NA \\
9 & 7.38 & 30.3 & 17.4 & 138 & 3.6 & 103 & 97 & 9.2 & 17.6 & 68.9 & NA & NA \\
Mean & 7.20 & 40.53 & 17.77 & 140 & 45 & 102 & 259 & 7.3 & 20.45 & 61.04 & NA & NA \\
(SD) & $(0.18)$ & $(24.79)$ & $(14.37)$ & $(11)$ & $(1)$ & $(5.6)$ & $(486)$ & $(6.0)$ & $(11)$ & $(55.52)$ & & \\
\hline
\end{tabular}

NA - not available; AG — anion gap; CK — creatine kinase; OG — osmolar gap; SD — standard deviation; SCr — serum creatinine

Table 3. Admission electrocardiogram characteristics.

\begin{tabular}{lcccccccc}
\hline Patient & Rhythm & $\begin{array}{c}\text { Axis } \\
{\left[{ }^{\circ}\right]}\end{array}$ & $\begin{array}{c}\text { HR } \\
{[\mathrm{bpm}]}\end{array}$ & $\begin{array}{c}\text { PR } \\
{[\mathrm{ms}]}\end{array}$ & $\begin{array}{c}\text { QRS } \\
{[\mathrm{ms}]}\end{array}$ & $\begin{array}{c}\text { OTc } \\
{[\mathrm{ms}]}\end{array}$ & T-wave & Comments \\
\hline 1 & Sinus & +90 & 115 & 144 & 98 & 428 & - & Patient required ventilation \\
2 & Sinus & +25 & 102 & 140 & 90 & 465 & Inversion $\mathrm{V}_{1}, \mathrm{~V}_{2}$ & - \\
3 & Sinus & +75 & 101 & 130 & 74 & 448 & - & - \\
4 & Sinus & +105 & 76 & 158 & 96 & 410 & - & $\begin{array}{c}\text { Admitted } 24 \mathrm{~h} \text { after } \\
\text { methanol ingestion }\end{array}$ \\
5 & Sinus & +73 & 83 & 160 & 100 & 435 & - & - \\
6 & Sinus & +84 & 115 & 128 & 88 & 445 & Peaked T-waves & Admitted 24 h after \\
7 & Sinus & -10 & 70 & 206 & 96 & 405 & Inversion III, aVF & methanol ingestion \\
8 & Sinus & +50 & 86 & 146 & 78 & 420 & Flat III, aVF & Fragmented ORS $\mathrm{V}_{3}-\mathrm{V}_{6}$ \\
\hline
\end{tabular}

On admission, these included sinus tachycardia (44\%), PR prolongation (11\%), $\mathrm{QT}_{\mathrm{c}}$ prolongation (22\%) and non-specific T-wave changes (66\%). Post-intoxication ECGs were available for 4 of the 9 patients, all of which demonstrated normalization of intervals and resolution of T-wave changes.

Patients with severe metabolic acidosis showed more significant ECG changes. Patient number 6 presented with a pH of 6.91 and his ECG (Fig. 1) showed sinus tachycardia $(\mathrm{HR}=115 \mathrm{bpm})$ with peaked T-waves in the precordial leads $\left(\mathrm{V}_{1}-\mathrm{V}_{4}\right)$ and the inferior leads. It should be noted, however, that this patient also had a potassium level of $5.9 \mathrm{mmol} / \mathrm{L}$. Patient number 2 presented with a $\mathrm{pH}$ of 7.09 and her ECG (Fig. 2) showed sinus tachycardia (HR = $=102 \mathrm{bpm}$ ) with $\mathrm{T}$-wave inversion in leads $\mathrm{V}_{1}$ and $\mathrm{V}_{2}$. There was also a mild $\mathrm{QT}_{\mathrm{c}}$ prolongation (463 ms).

While in hospital, 7 patients required hemodialysis, 3 required mechanical ventilation,
3 developed visual impairment, and 1 died (Table 4). Patient number 1 also developed a type- 1 Brugada pattern while he was concurrently sedated with an infusion of propofol, a sodium channel blocker known to potentially induce Brugada ECG pattern.

\section{Discussion}

Methanol intoxication and its possible effects on the cardiac conduction system have been scarcely reported in the literature. One case report documented a patient with methanol overdose that developed right bundle branch block in addition to left anterior fascicular block, paroxysmal atrial fibrillation and increased left ventricular end-diastolic and end-systolic dimensions [6]. In a case series of 8 men with methanol overdose published by Weisberger and Maclaughlin [7], ECG changes were observed in 7 men and these 


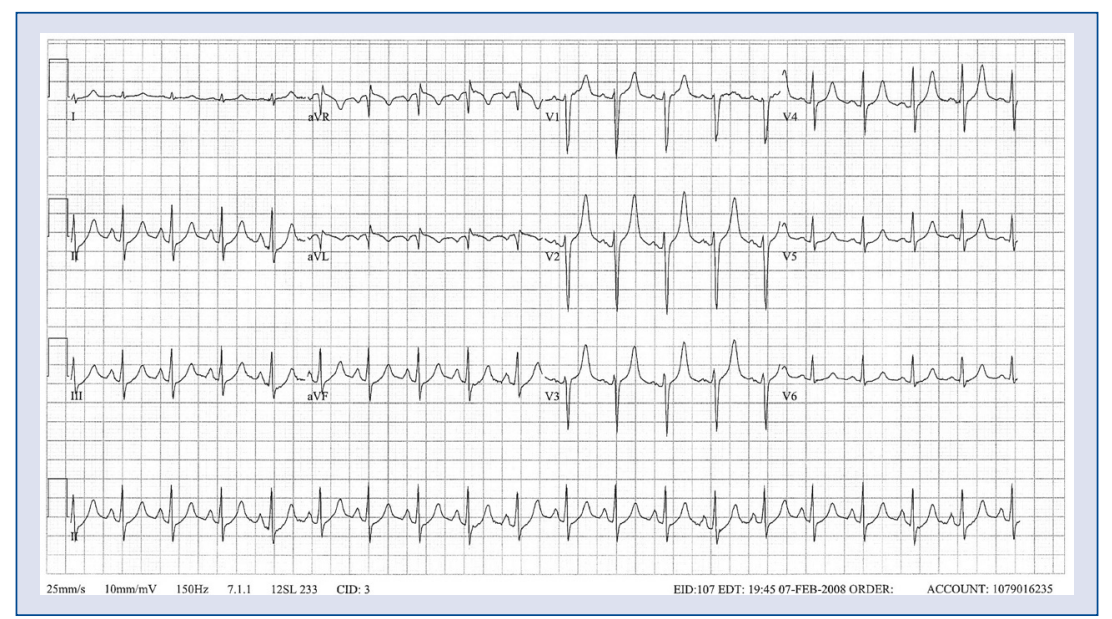

Figure 1. Patient \# 6: 12-lead ECG showing sinus tachycardia with peaked T-waves in the precordial leads $\left(V_{1}-V_{4}\right)$ and the inferior leads. P-wave electrical axis is shifted to the right, close to $+85^{\circ}$, voltage in the inferior leads $>2.5 \mathrm{~mm}$ indicating right atrial enlargement. Note that the peaked T-waves may be explained by concomitant mild hyperkalemia (potassium was $5.9 \mathrm{mmol} / \mathrm{L}$ ).

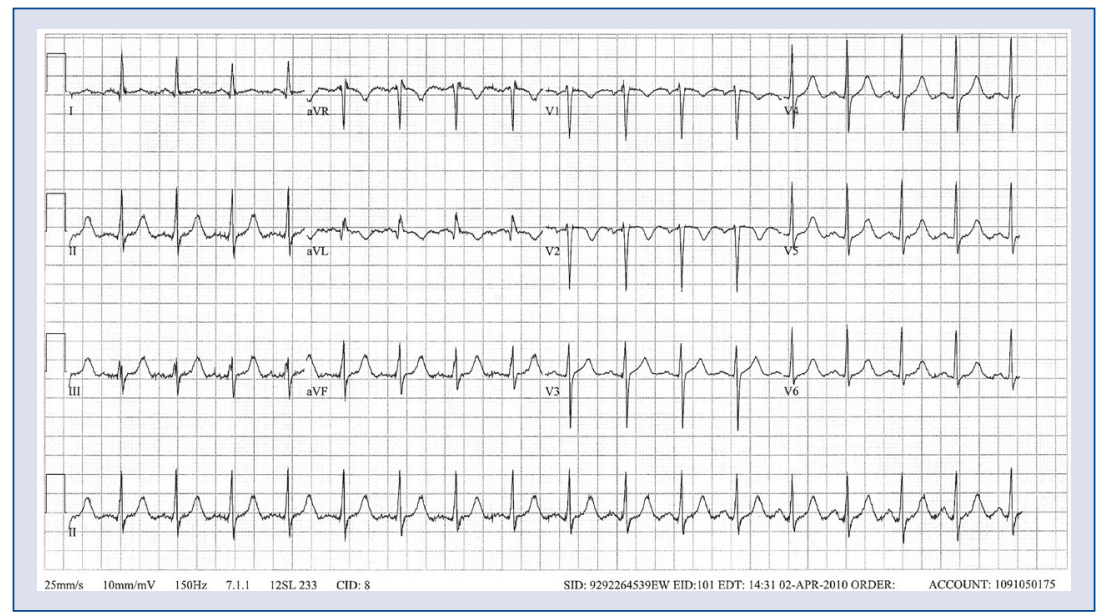

Figure 2. Patient \# 2: 12-lead ECG depicting sinus tachycardia with T-wave inversion in leads $\mathrm{V}_{1}$ and $\mathrm{V}_{2}$. Mild $\mathrm{QT}_{\mathrm{c}}$ prolongation (463 $\mathrm{ms})$.

Table 4. Clinical evolution and complications.

\begin{tabular}{lccccccc}
\hline Case & $\begin{array}{c}\text { Mechanical } \\
\text { ventilation }\end{array}$ & $\begin{array}{c}\text { Inotrope } \\
\text { support }\end{array}$ & Resuscitation & Dialysis & Death & $\begin{array}{c}\text { Length of } \\
\text { stay [days] }\end{array}$ & Complications \\
\hline 1 & Yes & No & No & Yes & Yes & 3 & $\begin{array}{c}\text { Permanent neurologic } \\
\text { damage }\end{array}$ \\
2 & No & No & No & Yes & No & 4 & $\begin{array}{c}\text { None reported } \\
\text { Visual Impairment } \\
3\end{array}$ \\
\hline & No & No & No & No & No & 5 & None reported \\
5 & No & No & No & Yes & No & 2 & None reported \\
6 & No & No & No & Yes & No & 1 & Blindness \\
7 & Yes & No & No & Yes & No & 6 & None reported \\
8 & No & No & No & No & No & 1 & Visual Impairment \\
9 & Yes & No & No & Yes & No & 6 & None reported
\end{tabular}


included low voltage T-waves in leads I and II, increased T-wave voltages in lead III and slightly prolonged QTc intervals. Another case series of 14 patients conducted by Hazra et al. [8], described right atrial overload with tall peaked $\mathrm{P}$-waves, clockwise rotation with a shift of transitional zone to the right, and right ventricular strain with tall $\mathrm{R}$-waves in the right precordial leads [8]. The regression of these ECG changes coincided with clinical improvement and suggests that they were caused by methanol poisoning.

In our study, clinical improvement and ECG normalization were also noted once methanol intoxication was resolved. However, due to the retrospective nature of the study design, no causality can be established between methanol intoxication and ECG changes. Furthermore, there is some disparity in our results as compared to what has been reported previously in the literature. The study by Cavalli et al. [6] focused on describing the possible relationship between methanol intoxication and cardiac dysfunction. As such, the authors reported some ECG changes as part of the description. They did not look specifically at the relationship between methanol and ECG changes. In other studies [7, 8], authors did not describe possible confounding variables such as substance co-ingestion, concurrent illnesses, pre-existing cardiac history and use of anti-arrhythmic medications. In the study reported by Weisberger and Maclaughlin [7], the results of ECG were provided relative to the time of admission with no additional details regarding the methanol level at the time as well as the degree of acidosis. Because of these differences, it is difficult to compare the results of our study with prior series.

ECG changes associated with methanol intoxication have been attributed to formic acid, a metabolite of methanol. A direct correlation between formic acid accumulation and methanol toxicity has been demonstrated [10], and may lead to increased morbidity and mortality [9]. Many factors can control the rate of formic acid metabolism in humans; the oxidation of formic acid is dependent on hepatic tetrahydrofolate concentrations, which depend on both adequate dietary folic acid and the efficiency with which tetrahydrofolate is regenerated [9]. As such, people with an adequate intake of folic acid may be protected from the toxic effects of formic acid and supplementation of folic acid may play a role in the treatment of methanol intoxication [5].

There are few hypotheses that attempt to explain the ECG changes observed during methanol intoxication. Methanol and formic acid can decrease the heart rate and the contractility of the myocardium [6]. Since formic acid can inhibit cytochrome oxidase activity in intact mitochondria, it has the potential to cause histotoxic hypoxia if the inhibition occurs at the terminal end of the respiratory chain [5]. The resulting sympathetic changes may trigger pulmonary hypertension and right atrial overload and right ventricular strain [6].

Although acidosis is well-documented during methanol intoxication, it is unclear whether it is derived from formic acid directly or due to its secondary effects of inducing lactic acidosis [11]. Independent of the etiology of the acidosis, it is well known that metabolic acidosis can lead to various adverse effects to the cardiovascular system. Cardiac contractility and cardiac output are reduced and arterial vasodilation develops, which may contribute to hypotension [12]. In animal models of metabolic acidosis, a predisposition to ventricular arrhythmia is often observed [12]. In 1 animal study with dogs, increased susceptibility of the heart to ventricular fibrillation was found during metabolic acidosis [13]. In another animal study with rats, the authors concluded that acidosis produced a marked decrease in heart rate and an increase in P-R interval with no apparent effect on the duration of the QRS complex [14].

It is also interesting to note that although acidosis appears to be consistently observed in methanol intoxication, an increase in anion gap or osmolality gap may not be observed consistently throughout the course of methanol intoxication. During the early course of methanol intoxication, a significant anion gap may not be apparent when the serum bicarbonate concentration falls in conjunction with a compensatory increase in the serum chloride concentration [5]. However, as the metabolism of methanol continues, a raised anion gap metabolic acidosis develops [5]. Likewise, the presence of an elevated osmolality gap suggests the presence of significant concentrations of methanol or other alcohols [5]. However, as methanol is metabolized to formic acid, the contribution of formic acid to osmolality gap becomes insignificant as formate is charged and electrically balanced by sodium. Therefore, the osmolality gap does not reflect the severity of methanol poisoning and the absence of the osmolality gap does not exclude the possibility of methanol intoxication [5].

Some patients did not develop acidosis, specifically patients 3,7 and 8 . Patients 3 and 7 presented to the hospital with relatively low methanol concentrations and neither had a raised anion gap, suggesting that these cases were not 
severe methanol intoxication. ECG changes were observed nevertheless; these changes might be attributable to the direct effects of methanol or formic acid independent of acid-base status.

In this study, the number of ECG changes seems to be related to the severity of the acidosis. A lower $\mathrm{pH}$ was associated with multiple ECG changes. This observation is possibly explained by an accumulation of formic acid contributing to worsening acidosis. The sinus tachycardia commonly observed in our patients was perhaps a nonspecific systemic inflammatory response to illness or stress. It is likely that the T-wave changes are a result of stress and/or non-specific repolarization abnormalities owing to disruption of the cellular milieu by acidosis. In addition, the increase in PR and QTc interval could be the direct action of methanol on conductivity, or a non-specific sympathetic response.

\section{Limitations of the study}

The retrospective nature of the report could introduce bias. In addition, no direct claims of causality can be made about the association between $\mathrm{pH}$ and ECG changes due to the difficulty of isolating its effects from concomitant biochemical aberrations or drug interactions. Moreover, it would have been desirable to have baseline and follow-up ECGs in all cases.

Serum folate levels were not available for analysis. However, it may be of interest to obtain folate levels in future prospective studies to determine if any relationships or patterns can be elucidated.

Finally, the small sample size is a limiting factor; however, given the observed changes, a larger prospective observational study may be warranted.

\section{Conclusions}

This study evaluated the effect of methanol on the human ECG. Although methanol intoxication led to several ECG changes that may have been dependent on the severity of the acidosis, no specific ECG findings or patterns could be detected.

Conflict of interest: none declared

\section{References}

1. McMahon DM, Winstead S, Weant KA. Toxic alcohol ingestions: Focus on ethylene glycol and methanol. Adv Emerg Nurs J, 2009; 31: 206-213.

2. Abramson S, Singh AK. Treatment of the alcohol intoxications: Ethylene glycol, methanol and isopropranol. Curr Opin Nephrol Hypertens, 2000; 9: 695-6701.

3. Megarbane B, Borron SW, Baud EJ. Current recommendations for treatment of severe toxic alcohol poisonings. Intensive Care Med, 2005; 31: 189-195.

4. Bronstein AC, Spyker, DA, Cantilena LR Jr. 2007 Annual report of the American Association of Poison Control Centers' National Poison Data System (NPDS): $25^{\text {th }}$ Annual Report. Clin Toxicol, 2008; 46: 927-957.

5. Barceloux DG, Bond GR, Krenzelok EP. American Academy of Clinical Toxicology Ad Hoc Committee on Treatment of Methanol Poisoning. American Academy of Clinical Toxicology Practice Guidelines on the treatment of Methanol Poisoning. J Toxicol Clin Toxicol, 2002; 40: 415-446.

6. Cavalli A, Volpi A, Maggioni AP. Severe reversible cardiac failure associated with methanol intoxication. Postgrad Med J, 1987; 63: 867-868.

7. Weisberger AS, Maclaughlin JA. Electrocardiographic changes associated with methyl alcohol poisoning. Am Heart J, 1947; 33: 27-33.

8. Hazra DK, Seth HC, Mathur KS et al. Electrocardiographic changes in acute methanol poisoning. J Assoc Physicians India, 1974; 22: 409-413.

9. Brent J. Fomepizole for ethylene glycol and methanol poisoning. N Engl J Med, 2009; 360: 2216-2223.

10. Tephly TR. The toxicity of methanol. Life Sci, 1991; 48: 1031-1041.

11. McMartin CE, Ambre JJ, Tephly TR. Methanol poisoning in human subjects. Role for formic acid accumulation in the metabolic acidosis. Am J Med, 1980; 68: 414-418.

12. Kraut JA, Madias NE. Metabolic acidosis: Pathophysiology, diagnosis and management. Nat Rev Nephrol, 2010; 6: 274-285.

13. Gerst PH, Fleming WH, Malm JR. Increased susceptibility of the heart to ventricular fibrillation during metabolic acidosis. Circ Res, 1966; 19: 63-70.

14. Aberra A, Komukai K, Howarth FC, Orchand CH. The effect of acidosis on the ECG of the rat heart. Exp Physiol, 2001; 86: 27-31. 